\title{
BMJ Open Pharmacist and Data-Driven Quality Improvement in Primary Care (P-DQIP): a qualitative study of anticipated implementation factors informed by the Theoretical Domains Framework
}

To cite: Tang J, Toma M, Gray NM, et al. Pharmacist and Data-Driven Quality Improvement in Primary Care (P-DQIP): a qualitative study of anticipated implementation factors informed by the Theoretical Domains Framework. BMJ Open 2020;10:e033574. doi:10.1136/ bmjopen-2019-033574

- Prepublication history and additional material for this paper are available online. To view these files, please visit the journal online (http://dx.doi org/10.1136/bmjopen-2019033574).

Received 14 August 2019 Revised 16 January 2020 Accepted 30 January 2020

D) Check for updates

(c) Author(s) (or their employer(s)) 2020. Re-use permitted under CC BY-NC. No commercial re-use. See rights and permissions. Published by BMJ.

For numbered affiliations see end of article.

Correspondence to Dr Tobias Dreischulte; tobias.dreischulte@med.unimuenchen.de

\section{ABSTRACT}

Objectives The quality and safety of drug therapy in primary care are global concerns. The Pharmacist and Data-Driven Quality Improvement in Primary Care (P-DQIP) intervention aims to improve prescribing safety via an informatics tool, which facilitates proactive management of drug therapy risks (DTRs) by health-board employed pharmacists with established roles in general practices. Study objectives were (1) to identify and prioritise factors that could influence P-DQIP implementation from the perspective of practice pharmacists and (2) to identify potentially effective, acceptable and feasible strategies to support P-DQIP implementation.

Design Semistructured face-to-face interviews using a Theoretical Domains Framework informed topic guide. The framework method was used for data analysis. Identified implementation factors were prioritised for intervention based on research team consensus. Candidate intervention functions, behavioural change techniques (BCTs) and policies targeting these were identified from the behavioural change wheel. The final intervention content and modes of delivery were agreed with local senior pharmacists.

Setting General practices from three Health and Social Care Partnerships in National Health Service (NHS) Tayside. Participants 14 NHS employed practice pharmacists. Results Identified implementation factors were linked to thirteen theoretical domains (all except intentions) and six (skill, memory/attention/decision making, behavioural regulation, reinforcement, environmental context/resources, social influences) were prioritised. Three intervention functions (training, enablement and environmental restructuring) were relevant and were served by two policy categories (guidelines, communication/marketing) and eight BCTs (instructions on how to perform a behaviour, problem solving, action planning, prompt/cues, goal setting, self-monitoring, feedback and restructuring the social environment). Intervention components encompass an informatics tool, written educational material, a workshop for pharmacists, promotional activities and small financial incentives.

Conclusions This study explored pharmacists' perceptions of implementation factors which could

\section{Strengths and limitations of this study}

- This qualitative study used a theory-driven and structured approach to identify factors that may influence the sustained implementation of medication safety intervention in primary care Pharmacist and Data-Driven Quality Improvement in Primary Care (P-DQIP).

- The design of the P-DQIP intervention combined consideration of theoretically underpinned strategies with knowledge of the local implementation context.

- The applicability of our findings may be limited to settings, in which National Health Service employed pharmacists have established roles within general practices.

- The prioritisation of theoretical domains may be biased by our previous experience of developing successful prescribing safety interventions in primary care.

- Intervention design that combines theory and experience limits the ability to test their respective contributions in driving behavioural change.

influence management of DTRs in general practices to inform implementation of P-DQIP, which will initially be implemented in one Scottish health board with parallel evaluation of effectiveness and implementation.

\section{BACKGROUND}

The quality and safety of medication use in primary care is an increasing concern in the UK and internationally. Up to $4 \%$ of all unplanned hospital admissions are caused by preventable adverse drug events (ADEs). ${ }^{1-5}$ Older people are particularly at risk of drug-related harm, because of their often increased susceptibility to ADEs and frequent use of polypharmacy, increasing the likelihood of drug therapy risks (DTRs) including drug-drug and drug-disease interactions. ${ }^{67}$ 
In parallel to population ageing, the prevalence of polypharmacy and DTRs is rising ${ }^{8}$ and so are drug-related hospital admissions ${ }^{9}$ as well as outpatient and emergency room visits. ${ }^{10}$

A number of recent UK trials have evaluated interventions, in which electronic medical records (EMRs) were used to identify and target patients with DTRs for review. The pharmacist-led information technology intervention for medication errors (PINCER) employed pharmacists for 12 weeks to identify and review patients with high-risk prescribing and monitoring from EHRs. ${ }^{11}$ In contrast, the data-driven quality improvement in primary care (DQIP) intervention ${ }^{12}$ was general practitioner (GP)-led and provided education and a small financial incentive to promote the use of an informatics tool, which identifies and facilitates review of patients with DTRs. Both trials demonstrated significant reductions in targeted DTRs. However, the impact of the PINCER intervention waned after withdrawal of pharmacist support, while reductions in targeted DTRs were sustained in the year after the DQIP intervention ceased (because it led to reduced initiation of high-risk prescribing by GPs) ${ }^{11}{ }^{12}$ Additionally, the DQIP trial provided some evidence of reduced hospital admissions linked to targeted DTRs. ${ }^{12}$

In spite of these encouraging findings, both DQIP and PINCER were limited by their relatively narrow scope (small number of targeted DTRs). Addressing the needs of older people on multiple drugs requires a much broader scope to encompass a range of indicators. For example, the Scottish government polypharmacy working group has developed 69 indicators to identify older people with DTRs linked to 18 ADEs (eg, falls and fractures, bleeding, hypoglycaemia) from EMRs. ${ }^{13}$ Inevitably, a broader scope will lead to identifying more patients needing review, which has resource implications. ${ }^{14}$ In response to the GP workforce crisis, all four UK countries are currently investing in new posts for pharmacists to work alongside general practice teams, with National Health Service (NHS) England investing £130 million for 2000 additional practice pharmacists. ${ }^{15-18}$

P-DQIP aims to implement and evaluate a DTR management intervention that is pharmacist and data driven and deliverable, scalable and sustainable in the UK's NHS. Given prior evidence of effectiveness, we plan to include in the intervention core elements from PINCER ${ }^{11}$ (pharmacist-driven approach) and DQIP ${ }^{12}$ (informatics tool to identify and review patients with DTRs). P-DQIP will target a broader range of DTRs relevant to older people, and rather than employing new pharmacists (as in PINCER), NHS-employed pharmacists already affiliated with general practices will work as part of practice teams in order to facilitate sustained impact.

The aim of this study was to systematically develop a theoretically informed strategy to support implementation of pharmacist (P-DQIP) in NHS Scotland. To this end, it is important to understand factors that may drive successful implementation. For the purposes of this study, we define implementation factors as characteristics of individuals or the environment they work in, which may influence the implementation of P-DQIP. The study objectives were (1) to identify implementation factors from the perspective of practice pharmacists and prioritise them for intervention and (2) to identify potentially effective, acceptable and feasible strategies to support P-DQIP implementation.

\section{METHODS}

\section{Theoretical framework and study design}

The study design draws on guidance on using the 'behavioural change wheel (BCW),19 (figure 1). The BCW is based on the capability, opportunity, motivation,

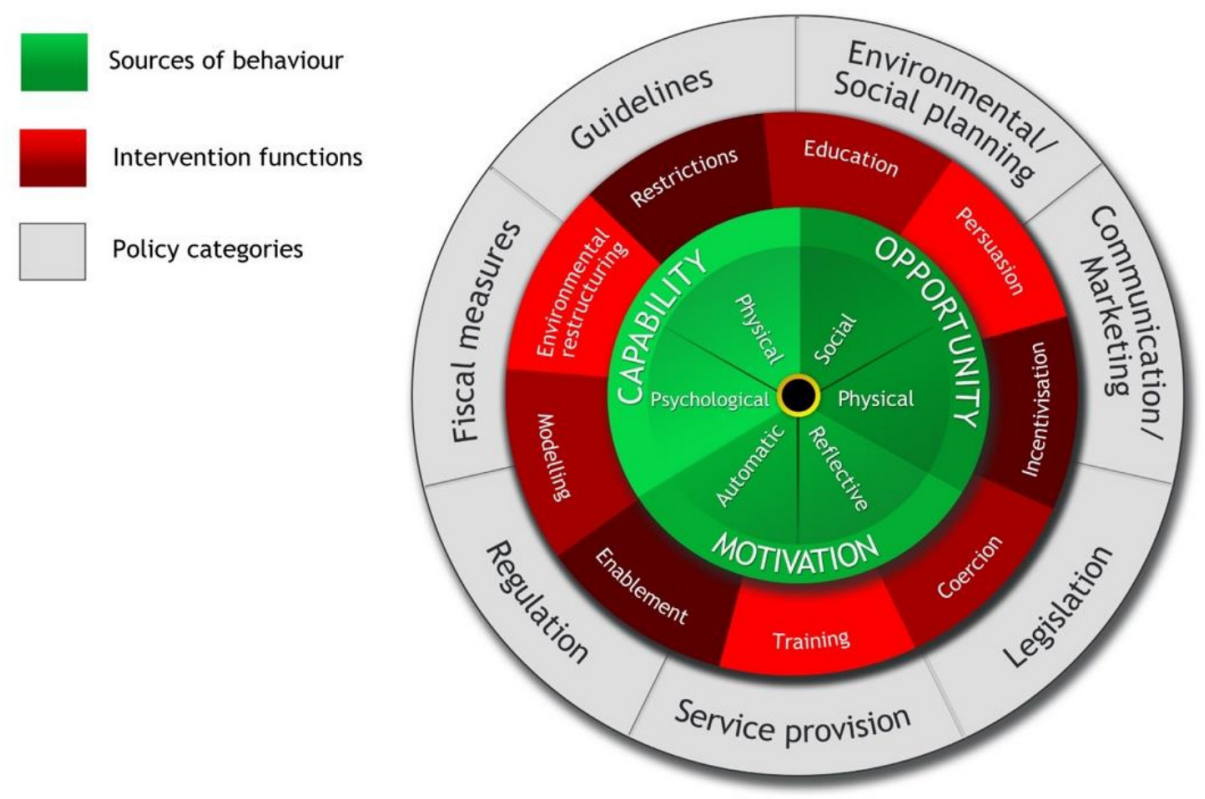

Figure 1 The behavioural change wheel. Reproduced from Michie and Atkins. ${ }^{19}$ 


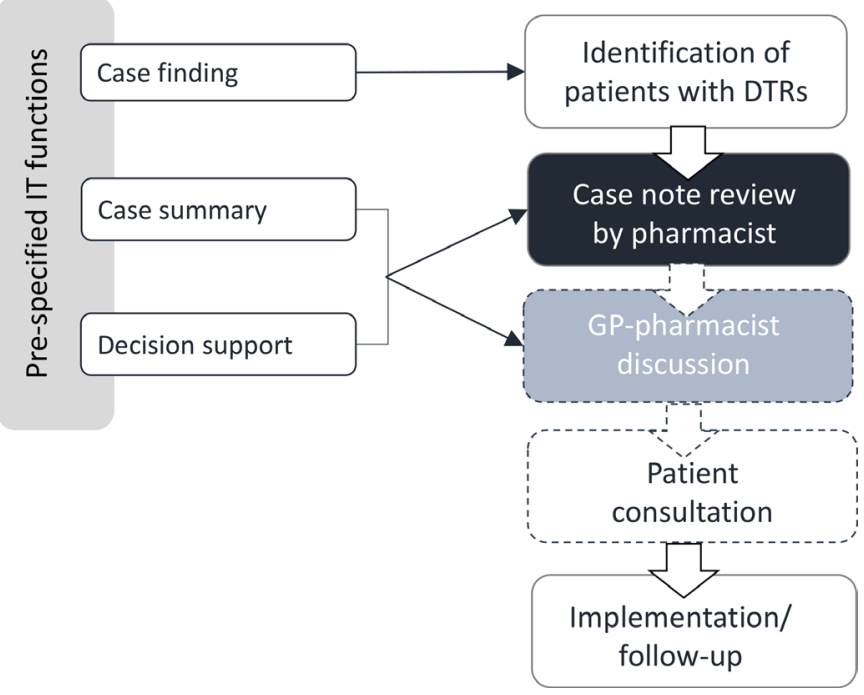

Figure 2 Intended drug therapy risk management model with behaviours to be targeted by the P-DQIP informatics tool. The dotted lines denote potential pathways, that is, pharmacists may decide on a DTR management strategy with or without prior consultation with patients or other clinicians. DTR, drug therapy risk; GP, general practitioner; IT, information technology; P-DQIP, Pharmacist and Data-driven Quality Improvement in Primary Care.

behaviour (COM-B) change model, which identifies six broad influences on behaviour (physical and psychological capability, social and physical opportunity, reflective and automatic motivation). The linked Theoretical Domains Framework (TDF) ${ }^{20}$ consists of 14 overarching domains providing a more granular analysis of the influences on behaviour. The BCW and TDF have been extensively used to design interventions targeting healthcare professionals' behaviour. $^{21} 22$

The intended process by which drug-related harm can be prevented in P-DQIP is shown in figure 2. Based on this, the behaviour to be targeted by the P-DQIP intervention was defined as pharmacists' management of DTRs identified by the P-DQIP tool. To achieve this, pharmacists need to accomplish the following key tasks: (1) make clinical decisions on whether and which medication changes are appropriate; (2) collaborate with other clinicians to agree and implement a DTR management strategy; (3) embed the P-DQIP work into their work routine. Most pharmacists would opportunistically conduct clinical medication reviews as part of their existing roles. However, proactively identifying patients with DTRs was a new element that was expected (as a minimum) to increase the volume of pharmacists' medication reviews and the frequency of pharmacist-GP interaction.

To address objective 1 , we conducted semistructured face-to-face interviews with practice pharmacists using an interview topic guide based on the TDF, and then prioritised TDF domains for intervention. For objective 2, we mapped candidate intervention functions (ie, mechanisms by which an intervention can change behaviour) to prioritised TDF domains using the 'BCW'. ${ }^{1923}$ We used this mapping to identify suitable intervention functions, behavioural change techniques (BCTs) (ie, the smallest 'active ingredients') and policies (ie, avenues through which an intervention is delivered $)^{23-25}$ via consensus discussion within the research team. We agreed the final intervention content and delivery formats with local stakeholders (one senior practice pharmacists from each of three locality teams).

\section{Subjects and setting}

NHS Tayside has a total of 64 general practices serving a population of 425000 residents with a median list size of 6415 (range 1796-13 044) patients across all practices. General practices are organised geographically into three Health and Social Care Partnerships (HSCPs) and 12 'clusters', each cluster comprising between two and eight practices, who meet regularly to discuss quality improvement work. Each practice has at least one practice pharmacist representative, normally working in more than one practice. Their roles in these practices vary, but usually include cost-saving work (eg, switching patients to less expensive but therapeutically equivalent medicines) as well as undertaking complex clinical medication review. We purposively sampled NHS employed practice pharmacists aiming to include pharmacists from each of the three HSCP (reflecting pharmacy management structure) and with a range of working experience as practice pharmacists (which we anticipated to influence perceptions of implementation barriers). The NHS Tayside health board approached a total of 18 practice pharmacists on our behalf by email (including participant information sheet) asking them to get in touch with the research team if they were interested in participating. Of the pharmacists approached, eight worked in HSCP 1, five in HSCP2 and five in HSCP 3. Twelve had more than 5 years working experience (reflecting larger numbers of pharmacists working in HSCP 1 and a disbalance towards more experienced practice pharmacists in NHS Tayside at the time of the study).

\section{Data collection}

The interview topic guide (see online supplementary additional file 1) was drafted using the 14 domains of the $\mathrm{TDF},{ }^{20}$ piloted with three practice pharmacists and optimised iteratively to address all TDF domains and to minimise multiple questions yielding similar answers. The two interviewers also exchanged experiences after each interview, and iteratively amended the topic guide as required.

Semistructured interviews were conducted by two postdoctoral research fellows (one male (JT), one female (MT)) with backgrounds in health psychology and previous experience in conducting semistructured interviews. The interviews were conducted between December 2016 and March 2017 and took place in the pharmacists' place of work. The researchers had no prior relationships with any of the participants. The interviewers started the interview by providing background on the aims of P-DQIP and prespecified components (including paper mock-ups 
of the core functionalities of the P-DQIP informatics tool, namely case finding of patients with high-risk prescribing and review facilitation). Participants' perceptions of P-DQIP implementation factors were subsequently explored using the topic guide where it was tweaked iteratively as needed after each interview (depending on the exchange of experiences between the researchers). Interviews were audio recorded and transcribed verbatim by a professional transcription service. The researchers also cross-checked a subsample of four transcripts alongside their audio recordings to ensure accuracy of transcribing. All audio recordings were stored securely in accordance with institutional policies.

\section{Data analysis}

Data analysis was conducted by the core research team (JT, MT and TD) using NVivo V.11 (for initial identification of relevant quotes) and MS Excel (for coding of quotes identified as relevant).

\section{Identifying implementation factors (objective 1)}

Following a familiarisation process, data analysis was conducted in four steps.

The first step applied the framework method ${ }^{21}$ using a deductive approach to code identified implementation factors in relation to COM-B and TDF coding. A coding guideline (see online supplementary additional file 2) was iteratively developed and applied by two coders (JT and MT). All quotes were then coded by both researchers using this guideline, with disagreements resolved by consensus discussion.

The second step inductively developed a coding frame to identify specific beliefs among quotes coded to each TDF domain in step one. The coding frame was subsequently applied independently by two researchers (TD and JT), and disagreements were resolved by discussion.

The third step used consensus discussion to identify 'expected barriers' to P-DQIP implementation. An expected barrier was defined as a hindrance to P-DQIP implementation, which the interviewed pharmacist described as likely to occur in his or her own practice (rather than merely describing it as a relevant factor).

The final step explored links between theoretical domains.

\section{Prioritisation of implementation factors and mapping of intervention components (objective 2)}

In order to prioritise theoretical domains to be targeted by P-DQIP, we considered (as a crude guide) for each P-DQIP implementation factor within each theoretical domain: (1) how often it was coded; (2) how many participants it was coded for; (3) how often it was identified as an 'expected barrier' and (4) how feasible it was to address it as part of the P-DQIP intervention.

The prioritised theoretical domains were mapped onto components of the BCW. ${ }^{20}$ Apart from being potentially effective, prospective intervention components needed to be acceptable and feasible in the existing NHS context, which meant they had to be (1) deliverable by existing NHS staff with minimal training; (2) deliverable with minimal disruption to primary care clinicians' routine work and (3) involve minimal cost to the NHS. These criteria guided a stepwise review of potential intervention components by the core research team (JT, MT and TD), in which we first identified suitable intervention functions among those mapped to each TDF domain in Michie et $a l$ s mapping matrix. ${ }^{23}$ We then used the matrices linking intervention functions to policy categories ${ }^{24}$ and intervention functions to BCTs. ${ }^{25}$ Through consensus discussion, which involved practitioners with substantial experience in the health service, ideas on how to address identified barriers were explored until a consensus was reached before potential delivery mechanisms were agreed and intervention components drafted. The draft was subsequently presented to three HSCP pharmacy leads in a face-to-face meeting, where the final intervention components and delivery formats were then finalised.

\section{Patient and public involvement}

Feedback on the P-DQIP implementation strategy was sought from two public self-selected representatives from NHS Tayside who had an interest in research on polypharmacy and risky prescribing within primary care. They attended research team meetings to advise on the project identifying which components would benefit patients the most based on their own experiences of polypharmacy.

\section{RESULTS}

\section{Participants}

Fourteen of the 18 pharmacists approached were recruited (4 did not reply after two reminders). Participants worked in practices in HSCP $1(n=7)$, HSCP $2(n=5)$ and HSCP $3(n=2)$. Most pharmacists $(n=11)$ had over 5 years experience as practice pharmacists and most $(n=10)$ worked in two or more practices. Two researchers (MT and JT) conducted seven face-to-face interviews each and interviews lasted from 30 min to 1 hour. Data saturation was reached after 12 interviews. The additional interviews were conducted before the point of data saturation was established.

\section{Identified implementation factors (objective 1)}

A total of 211 quotes (ie, pieces of text judged as part of the same argument or thought) were identified as relevant to the target behaviour. The quotes represented 13 of the theoretical domains (all except 'intentions', which were defined as explicit expressions of commitment or lack thereof, which were less likely given that the intervention was hypothetical at the point of interview) and encompassed five COM-B constructs (all except 'physical skills') (see table 1 for sample quotes). In the following, we report findings organised by key pharmacist implementation tasks as outlined above, namely: (1) clinical decision making; (2) collaboration with other clinicians and (3) embedding the P-DQIP work in work routines. 


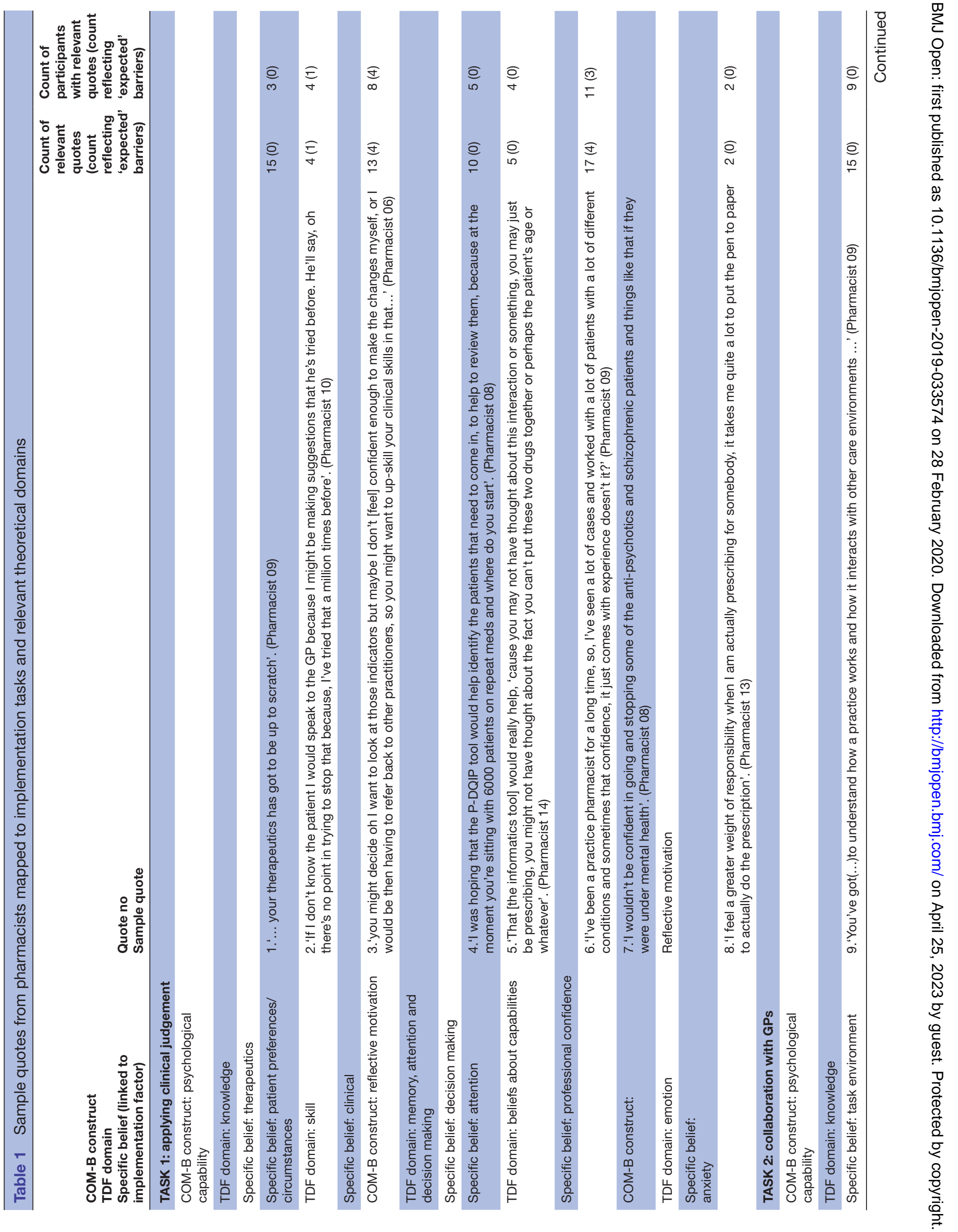




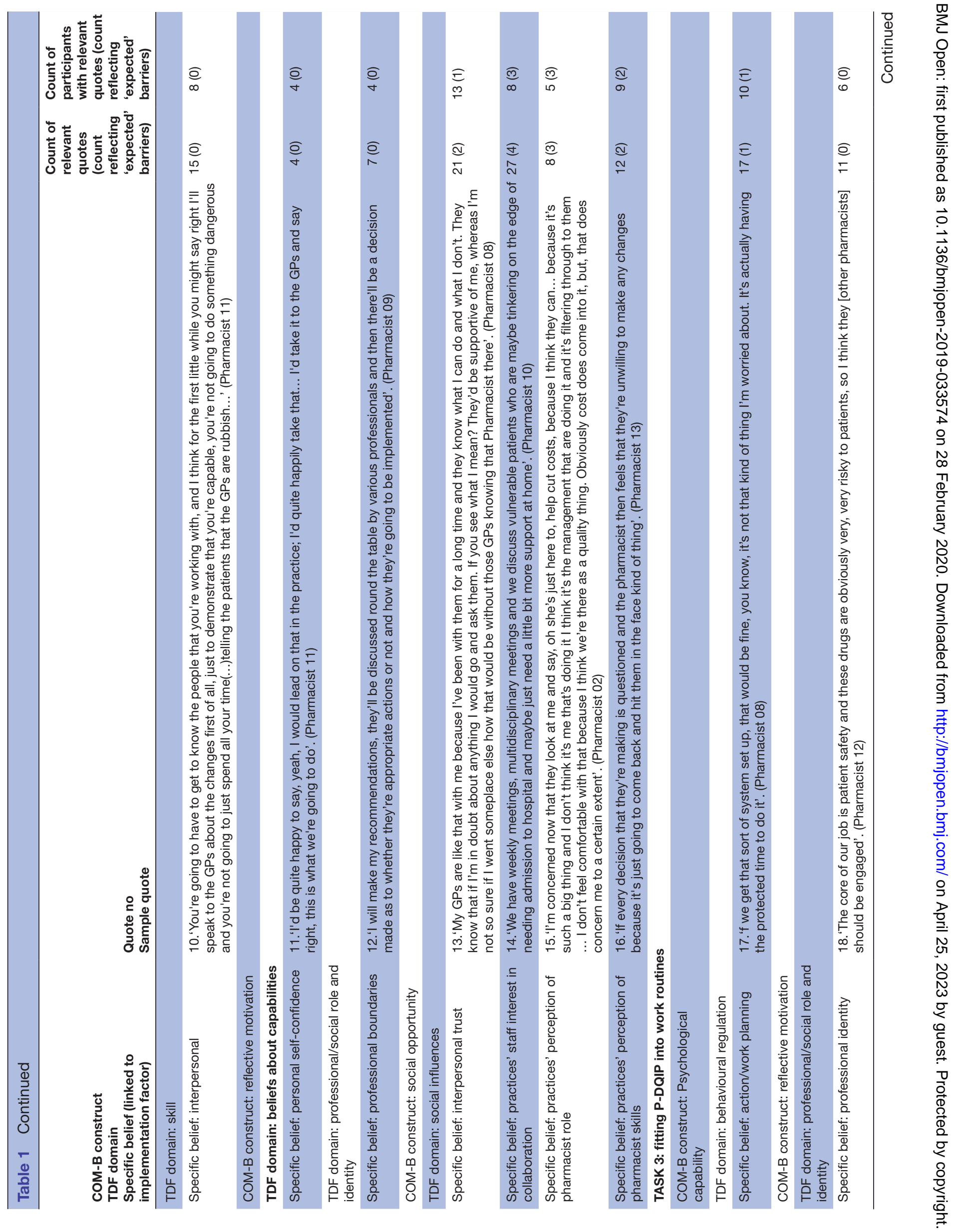




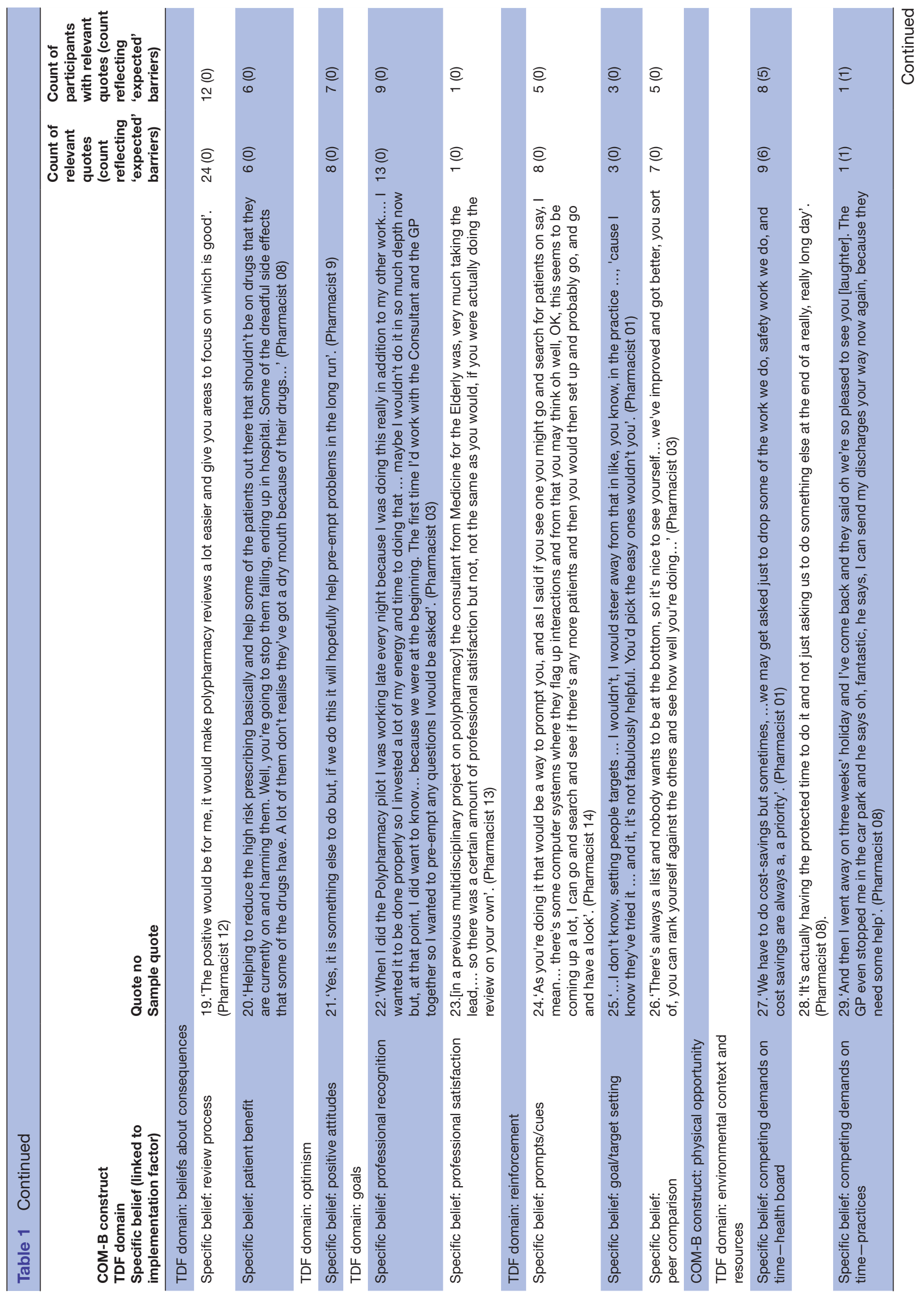




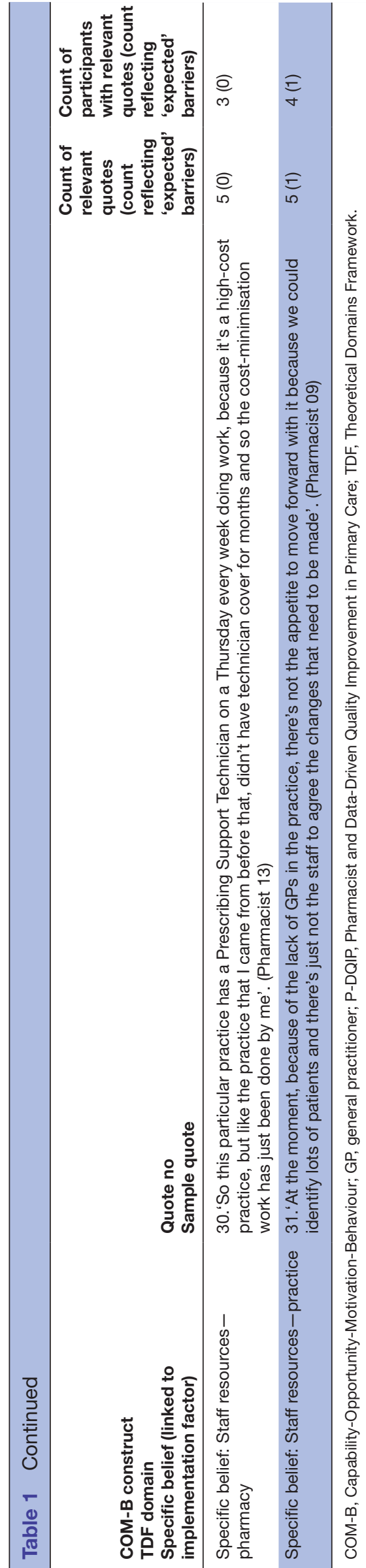

Task 1: clinical decision making

Most pharmacists identified up-to-date pharmacotherapeutic knowledge (knowledge; quote 1) as being essential to managing DTRs appropriately. Although participants generally felt their undergraduate education equipped them with the necessary knowledge and skills, some highlighted the need for selective 'upskilling' to manage infrequent/unfamiliar DTRs (skills; quote 3). Having been shown the functionalities of the informatics tool, pharmacists expressed that it could help direct attention to DTRs, which may otherwise be overlooked (memory, attention and decision making; quote 5). Pharmacists' confidence in clinically managing DTRs appeared strongly associated with relevant experience of working as a practice pharmacist (beliefs about capabilities, quote 6). However, several pharmacists identified complex therapeutic scenarios that required discussion by the wider multidisciplinary team irrespective of experience and skill (beliefs about capabilities; quote 7). Some pharmacists felt their limited knowledge of patients' personal circumstances prevented them from making decisions on DTRs independently (knowledge, quote 2). Several pharmacists highlighted their role was to advise or recommend a course of action to manage DTRs, but that the ultimate decision lay with other professionals (professional/social role and identity; quote 12). A few pharmacists expressed anxiety about making certain clinical decisions independently (emotion; quote 8), while others highlighted that such independence could lead to a higher level of professional satisfaction (goals; quote 23).

\section{Task 2: collaboration with other clinicians}

Most felt that for pharmacists to lead on DTR management in a particular practice, familiarity with practice processes and systems (knowledge, quote 9 ) was essential. Interpersonal skills (skills, quote 10) and actual experience of working with other clinicians in the practice (social influences, quote 13) was seen as crucial to win or maintain their trust. Personal self-confidence (as a character trait) was deemed important when engaging with other clinicians in managing DTRs (beliefs about capabilities, quote 11). Some pharmacists reported to have accomplished a good working relationship with other clinicians in the practice (social influences; quotes 13, 14). However, despite efforts to integrate with practice teams, others felt that GPs perceived their primary role as a resource for cost-cutting (social influences; quote 15) and that GPs scepticism about their clinical skills (social influences, quote 16) were barriers to pharmacist-GP collaboration in DTR management. Feelings of frustration on misconceptions of the pharmacists' role (social influences, quote 15) and lack of trust in their capabilities by practice staff were evident (social influences, quote 16). Some believed that GP staff shortages limited opportunities to discuss and agree on strategies on how best to manage DTRs (environmental context and resources; quote 31 ). 
Task 3: embedding the P-DQIP work into work routines

Most pharmacists appeared motivated to implement the P-DQIP work. Several pharmacists expressed that medication reviews and patient safety were strongly aligned with their professional identity (professional/Social Role and Identity; quote 18), and valued the P-DQIP work as a potential means to achieve further recognition as clinicians by the wider primary care team (goals; quote 22). Half also expected tangible clinical benefits for patients (beliefs about consequences; quote 20). Most pharmacists were positive that using the P-DQIP informatics tool would support them in managing DTRs, despite expectations of increased workload (Optimism; quote 21). Although the majority of pharmacists thought that the informatics tool would make the review process more efficient and structured (quote 19), most expected difficulties in fitting review of patients proactively identified by the P-DQIP informatics tool into their work routines. A prominent theme was the perceived high workload of routine tasks (environmental context and resources; quotes 27-29), including cost-saving projects and medicines reconciliation after hospital discharge, which could at least partly be delegated to pharmacy technicians (environmental context and resources; quote 30 ).

As strategies to engage pharmacists in the P-DQIP work, some mentioned there was a need for protected time (quote 17). Several pharmacists expected that prompts to specific DTRs delivered by the P-DQIP tool during reviews could encourage them to address such DTRs systematically at practice level (reinforcement; quote 24). Feedback and peer comparison (eg, on reductions in targeted prescribing) were viewed favourably as strategies to encourage and maintain pharmacists' engagement (quote 26). In contrast, purely quantitative targets set by line managers (eg, a minimum number of reviews per week) were viewed as a disincentive to address DTRs that are more complex or time consuming to manage (quote 25).

\section{Interactions between TDF domains}

While participants generally believed pharmacists' professional skills (knowledge and skills, professional role and identity) enabled them to undertake the P-DQIP work, self-perceived levels of capability (beliefs about capabilities) varied depending on experience. Although pharmacists expressed a personal and/or professional desire to engage in the P-DQIP work (beliefs about consequences), fitting the work into their routines (behaviour regulation), was limited by competing demands on pharmacists' time imposed by NHS line managers and practices (environmental context and resources). Nevertheless, pharmacists believed that the informatics tool could make the process of identifying and reviewing DTRs more efficient (beliefs about consequences) and effective (memory, attention and decision making). In terms of social influences, practices' expectations of the pharmacists' role (clinical vs cost cutting work), their skills, and the level of trust in their profession and as individuals were all reported to influence beliefs about capabilities. These could also be limited by a lack GP availability to agree on medication changes (environmental context and resources), which in turn may be driven by GPs' competing demands and/ or a lack of interest in DTR management and/or collaboration with pharmacists (social influences).

\section{Prioritisation of theoretical domains and mapping of intervention components (objective 2)}

Table 2 shows theoretical domains prioritised and not prioritised for intervention for each of the three key pharmacist tasks as above. In the following, we outline the rationale for prioritisation and the selection of intervention functions, BCTs and policies for prioritised theoretical domains for each task.

\section{Task 1: clinical decision making}

In order to support pharmacists' capability to make appropriate clinical decisions, we prioritised the TDF domains skill, memory/attention/decision making and reinforcement. The prespecified functionalities of the P-DQIP informatics tool included case-finding patients with DTRs (BCT: prompts/cues targeting memory/ attention/decision making) as well as prompts to specific DTRs in individual patients (BCT: prompts/cues targeting reinforcement). In order to address varying clinical skills by pharmacist and by DTR, it was decided that we would supplement DTR prompts by brief guidance on the management of each DTR within the P-DQIP informatics tool and provide more detailed evidence and guidance around targeted DTRs in an accompanying manual (BCTs: instructions on how to perform a behaviour targeting skill). Although potentially more effective, we considered it unfeasible to provide (and for pharmacists to attend) comprehensive face-to-face education and training for the broad range of targeted DTRs within currently available resources.

\section{Task 2: collaboration with other clinicians}

A key rationale for designing a pharmacist-driven intervention was to enhance the capacity of primary care teams to systematically manage DTRs. A prerequisite to realising such benefits is that GPs are willing to delegate DTR management tasks to pharmacists, which depends on trust. On the other hand, some involvement of GPs in DTR management continues to be required, not least because of legal constraints (not all pharmacists are licensed prescribers). The discrepancies between their own and GPs' perceptions of their professional role reported by some pharmacists and a lack of trust in their clinical skills and capability are potential barriers to P-DQIP implementation, and so is a lack of engagement of GPs in DTR management and interest in collaboration with pharmacists. While P-DQIP could increase opportunities to develop trust, it is highly unlikely that a single intervention will change such perceptions much. To begin to promote collaborative working between GPs and practice pharmacists 

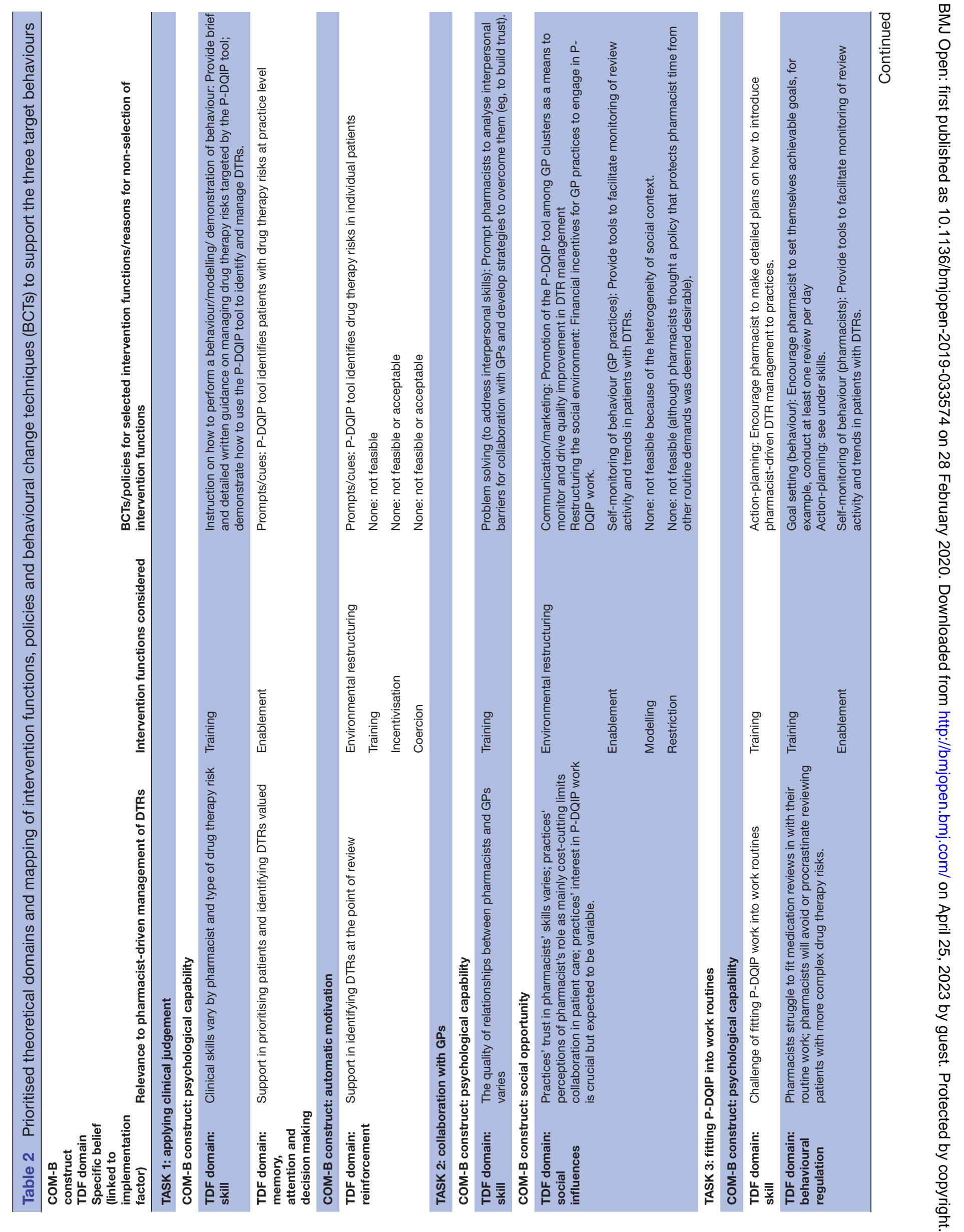

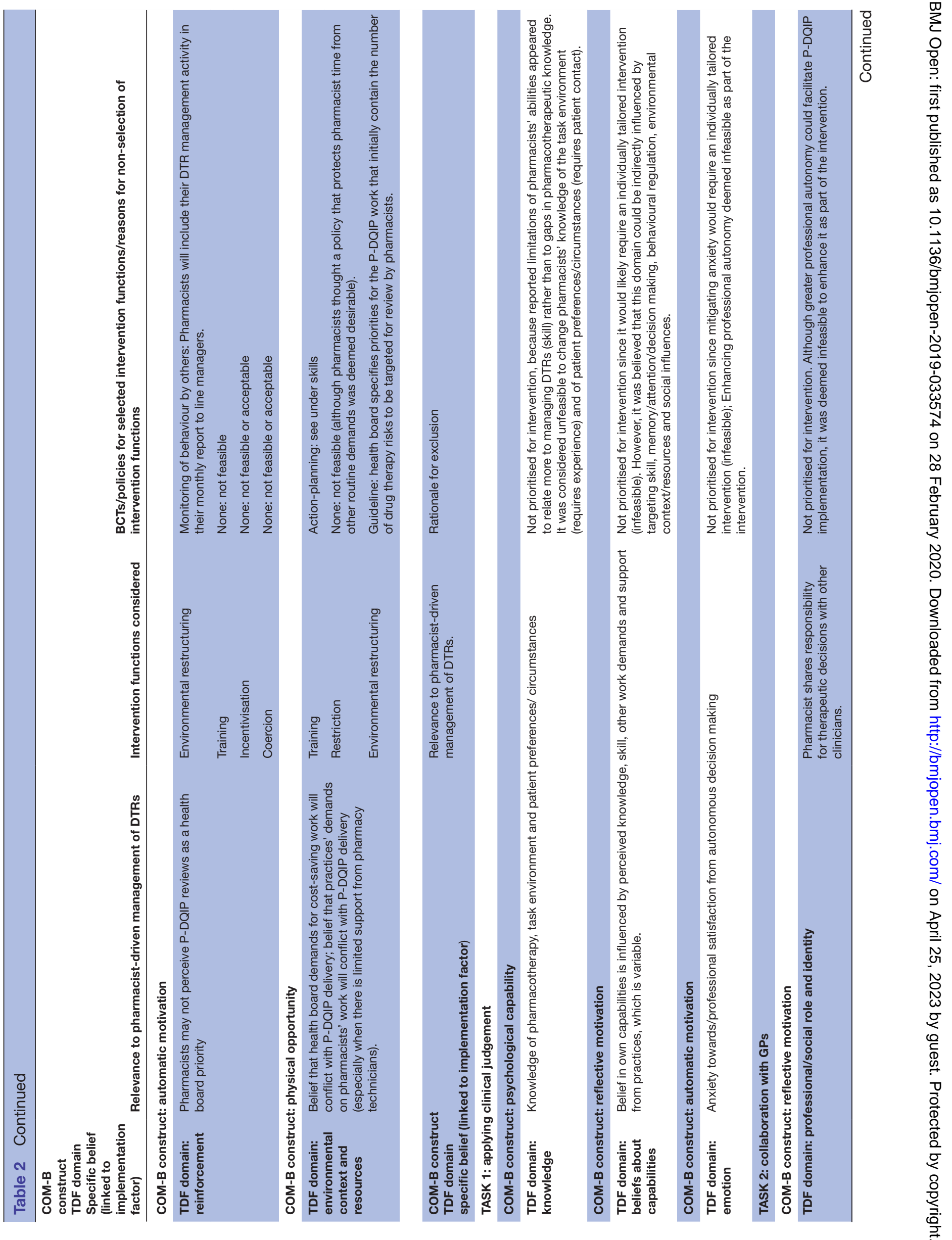
in general practices, we considered formal interprofessional education and action planning exercises. However, additional costs (GP locum fees), and poor attendance because of current GP staff shortages may prevent them from successful implementation. We have, therefore, decided to target pharmacists and GPs separately. We plan to support pharmacists by providing a platform for more experienced pharmacists to provide support to their peers. Pharmacists affiliated with practices in the same cluster will therefore participate in a moderated workshop to reflect on potential implementation barriers in their own settings and jointly develop strategies to overcome them, including an analysis of interprofessional or interpersonal barriers and strategies to engage GP's and win their trust (BCT: problem solving targeting skill). Additionally, we plan to engage GPs via strategies that have been successfully applied in the GP-led DQIP intervention, ${ }^{26}{ }^{27}$ namely encouraging practices to use the P-DQIP tool for systematic DTR management (policy: communication/marketing targeting social influences), provision of a web-based tool to enable practices to monitor trends in patients with targeted DTRs over time (BCT: self-monitoring targeting social influences) as well as small financial incentives (BCT: restructuring the social environment targeting social influences). Rather than an unconditional participation fee (as in DQIP), payment (GBP 450 ) in P-DQIP will be conditional on practices (1) nominating a lead GP for P-DQIP, (2) providing evidence of a meeting between the lead GP and practice pharmacist, in which a strategy for initiating and maintaining the practice's engagement in the P-DQIP work is agreed and (3) providing evidence of using the $\mathrm{P}-\mathrm{DQIP}$ tool to monitor their progress in reviewing and reducing targeted prescribing.

Task 3: embedding the P-DQIP work into pharmacists' work routines

The competing demands on pharmacist time inflicted on pharmacists by their health board employers as well as practices were a prominent theme in pharmacist interviews. A health board policy protecting pharmacist time for the P-DQIP work (as requested by one pharmacist) proved unfeasible. As an alternative, we aim to support pharmacists through training in time management (BCT: action-planning targeting skill) and goal setting (BCT: goal setting targeting behavioural regulation) as part of a moderated workshop (please see 'Task 2: collaboration with other clinicians' for further details). Since we expect the number of patients with DTRs identified by the informatics tool to exceed those manageable in a single effort, a stepwise approach to implementation will be adopted, where a smaller number of DTRs will be targeted initially in order to support pharmacists in planning and structuring the workload over time (policy: guideline targeting environmental context and resources). In order to maintain pharmacists' engagement over time, we will additionally 

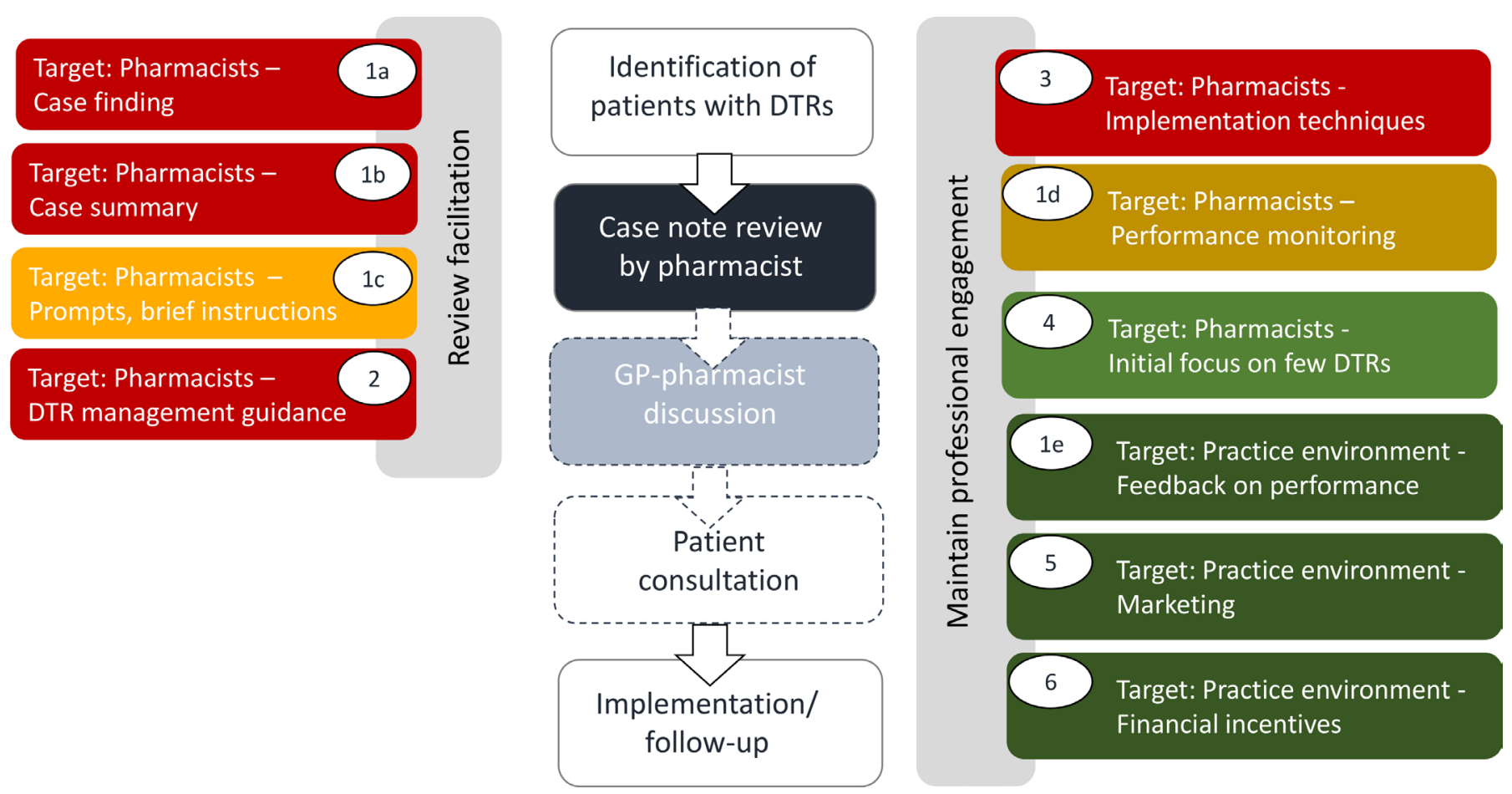

Figure 3 Final components of the P-DQIP intervention. Components are colour coded in terms of which COM-B category they primarily target (red: psychological capability; yellow: automatic motivation; amber: reflective motivation; light green: physical opportunity; dark green: social opportunity). Delivery mechanisms and content are numbered and specified in text below. (1) P-DQIP informatics tool integrated into existing Medicines management software (Scottish Therapeutics Utility): (1a) Search engine to identify patients triggering 18 composite and 69 individual indicators of drug therapy risks; (1b) Structured summaries of a patient's ongoing medical problems, investigations and current medications; (1c) Highlighting of a patient's identified drug therapy risks and brief management instructions; (1b) Facility to run weekly reports on the number of medication reviews submitted via the P-DQIP tool, with further details on medication changes, follow-up actions and time taken; (1e) Web-based application allowing practices to compare levels and trends of targeted prescribing to practices in their 'cluster', their HSCP and the health board. (2) Written educational material providing referenced evidence and guidance around targeted prescribing. (3) Half-day workshop with pharmacists affiliated with practices in the same 'cluster', moderated by P-DQIP lead pharmacists. Introduction of action planning instruments, goal setting and opportunity for pharmacists to discuss anticipated implementation problems and solutions. (4) Phased implementation of the review work with initial focus on patients at increased risk of a small number of specific adverse drug events. (5) Request to attend routine meetings of GP clusters by P-DQIP lead pharmacists to promote the use of the P-DQIP informatics tool to identify and facilitate the review of patients with DTRs and to monitor progress towards reducing targeted prescribing at practice and cluster levels. (6) Offer of payment of $£ 450$ per practice, which is conditional on providing evidence of conducting the following tasks: (1) Nominate a GP-lead for P-DQIP; (2) GP-pharmacist meeting to assign roles and responsibilities in P-DQIP work; (3) ongoing support for pharmacists in managing DTRs identified by the P-DQIP tool; (4) number of patients with DTRs reviewed by the practice over the P-DQIP intervention period. COM-B, Capability-Opportunity-Motivation-Behaviour; DTR, drug therapy risk; GP, general practitioner; HSCP, Health and Social Care Partnership; P-DQIP, pharmacist and data driven quality improvement in primary care.

provide information technology (IT) functionality to facilitate self-monitoring of review activity and trends in the numbers of patients with targeted DTRs (BCT: selfmonitoring of behaviour targeting behavioural regulation). In order to emphasise the importance that health boards assign to DTR management, line managers will request that pharmacists report on their DTR management activity on a monthly basis as they are currently doing for other routine tasks (BCT: monitoring of behaviour by others targeting reinforcement).

Figure 3 shows a diagram depicting the design of the P-DQIP intervention, which in the broadest terms comprises two elements aiming to (1) facilitate the identification and review of patients with DTRs and (2) maintain professional engagement and collaboration in this process.

\section{DISCUSSION}

\section{Summary of findings}

We systematically explored key implementation of a data and pharmacist-driven DTR management system (P-DQIP) from the perspective of NHS employed pharmacists (who will implement the intervention). Despite drawing on previously tested intervention components (an informatics tool with core functionalities (DQIP trial), and a pharmacist-led review model (PINCER trial)), we anticipated likely implementation and adoption 
challenges arising from the broadened scope and number of targeted patients, and from altering and/or adding to the work of NHS employed pharmacists with established roles in general practices. Consistent with the Medical Research Council complex interventions framework, ${ }^{28}$ we, therefore, considered it essential to better understand such challenges and identify potential strategies to address them before embarking on a wider implementation and evaluation of effectiveness. We used the TDF to comprehensively examine factors that could mediate (ie, support or hinder) P-DQIP implementation. ${ }^{20}$ Pharmacists felt that the core functionalities of the P-DQIP IT tool could address barriers relating to memory/attention and decision making (via prompts/cues). However, additional BCTs and/or policies were judged necessary to overcome barriers relating to five other TDF domains (skills, behavioural regulation, reinforcement, environmental context and resources, and social influences). Based on the interview data, these intervention components had the potential to positively influence pharmacists' beliefs in their capabilities, which were found to be key to the implementation of a DTR management system that is pharmacist driven.

\section{Comparison to previous research}

The notion that pharmacists are an underused clinical resource has stimulated a considerable amount of research on pharmacist-led interventions in primary care. Most previous evaluations, however, focus on 'nondispensing' or 'cognitive' services delivered by community pharmacists (as opposed to practice pharmacists employed by a healthcare funder, such as the NHS) and the design of interventions found in such evaluations are rarely explicitly theory based..$^{29}$ An exception is a qualitative study by Cadogan $e t a l,{ }^{22}$ in which the authors used the TDF-similar to this study-to guide selection of intervention components targeting prescribing (by GPs) and dispensing (by community pharmacists) for older people with polypharmacy in primary care. The theoretical domains prioritised for intervention by Cadogan et al broadly match the ones selected in this study, and all but one BCT ('social processes of encouragement/support') selected by Cadogan et al were also selected by us. Nevertheless, a noteworthy difference is our prioritisation of reinforcement, which reflects our intention to facilitate the sustained implementation of the P-DQIP intervention in NHS Scotland, rather than designing an intervention for evaluation in a randomised controlled trial. For the same reason, we additionally include a number of BCTs and locally agreed policies to encourage and maintain pharmacist-driven DTR management (goal setting, selfmonitoring of behaviour, monitoring of behaviour by others, promotion/marketing, guidelines). A further difference is our focus on informatics tools as a delivery mechanism for BCTs (cues/prompts, education, feedback), which was enabled by our opportunity to build on medicines management software which is available in all
Scottish GP practices, and which can interrogate practices' EMRs and generate reports.

\section{Strengths and limitations}

This study uses a theory-based systematic approach to design a strategy to support the implementation of a pharmacist-driven DTR management process in UK general practice. By describing our stepwise approach, starting with the specification of the target behaviours, identification and prioritisation of implementation factors, and finally, the selection of BCTs and policies, we provide complete transparency in our choice of intervention components optimising them for effective implementation. We used the framework method applying the widely used COM-B and TDF in conjunction with a systematic coding process, which was produced from a subset of the interviews. Further, we collaborated closely with local stakeholders to ensure that intervention components were feasible, acceptable and deliverable by existing NHS staff.

The main limitation of the study is that our findings may only apply to the context in which it was conducted, and therefore, may not represent the perspectives of practice pharmacists in general. Specifically, all interviewed pharmacists had established roles within their affiliated practice(s) and most had more than 5 years of experience working as practice pharmacists meaning that perspectives could be different in contexts where primary care pharmacy is less well established. Nevertheless, the proactive identification, review and management of DTRs as the target behaviour of the P-DQIP intervention, was novel to all participants, and we identified implementation barriers (eg, practices' trust in pharmacists' skills) that would be expected to be more prominent among pharmacists with less working experience in general or in the practices they work in. It is also possible that the prioritisation of theoretical domains and selection of intervention strategies was biased by our previous experience of developing successful prescribing safety interventions in primary care. ${ }^{12}$ However, by systematically considering intervention strategies based on mapping recommendations, we minimised the risk of omitting relevant theoretically underpinned alternative or additional strategies. Furthermore, the theoretical domains prioritised for intervention in this study broadly matched those identified in a similar study targeting community pharmacists, ${ }^{22}$ which, taken together, affirms their relevance to current policies which aim to extend pharmacists' clinical roles in primary care. Although the intervention design draws on an enhanced local infrastructure, implementing the IT components of the intervention would be possible (in principle) in any healthcare setting, where electronic health records are used. The study used experiential alongside theoretically underpinned design of intervention strategies. Although a pragmatic approach to intervention development, we acknowledge that it limits the ability to examine the respective contributions of theory and experience in driving behavioural change. 


\section{CONCLUSIONS}

The findings of this study are of particular relevance to the UK context, which has recently seen substantial investment in practice pharmacist posts to improve medicines management and reduce GP workload. Our study suggests that pharmacists' belief in their capabilities is a key factor influencing their capacity to extend their clinical roles, and this was, in turn, limited by their existing skill sets, available resources (including managing time in the face of conflicting demands), and underdeveloped working relationships with GPs.

The design of the optimised P-DQIP implementation strategy demonstrates that providing tools and training pharmacists alone is likely to be insufficient to sustain pro-active identification and management of patients with DTRs by teams of pharmacists and GPs. Aligning pharmacists' roles with the stepwise attainment of measurable practice-level performance goals may be one way of stimulating and maintaining concerted action by these professionals.

More broadly, comparison of the optimised P-DQIP intervention to an intervention with similar objectives developed by Cadogan et al highlights that intervention design choices are influenced by local implementation challenges as well as local opportunities to address them. While this may compromise the applicability of evaluation findings in other healthcare contexts, process evaluations can be used to help understand both the relative importance of intervention components and their interactions with local implementation context.

The P-DQIP intervention will be implemented in all practices in one NHS Scotland health board and evaluated in an interrupted time series study with parallel process evaluation to examine its implementation and effectiveness.

\section{Author affiliations \\ ${ }^{1}$ Scottish Improvement Science Collaborating Centre (SISCC), School of Nursing and Health Sciences, University of Dundee, Dundee, UK \\ ${ }^{2}$ Physiotherapy Department, NHS Tayside, Dundee, UK \\ ${ }^{3}$ Usher Institute of Population Health Sciences and Informatics, The University of \\ Edinburgh, Edinburgh, UK \\ ${ }^{4}$ Population Health Sciences Division, Medical Research Institute, University of Dundee, Dundee, UK \\ ${ }^{5}$ School of Nursing and Midwifery, Robert Gordon University, Aberdeen, UK \\ ${ }^{6}$ Health Services Research Unit, University of Aberdeen, Aberdeen, UK \\ ${ }^{7}$ Institute of General Practice and Family Medicine, University Hospital of Ludwig- \\ Maximilians-University, Munchen, Bayern, Germany}

\section{Twitter Madalina Toma @mada_toma84 and Aileen Grant @aileenmgrant}

Acknowledgements The listed order of the authors represents extent of contribution. The authors would like to thank the participating practice pharmacists, the three senior pharmacists who assisted in specifying the implementation strategy, and Suzanne Grant, who helped develop the topic guide and advised on data-analysis.

Contributors JT, MT and TD designed the study and conducted the qualitative analysis supported by JD. JT and TD jointly wrote the first draft of the manuscript, with all remaining authors, MT, NMG, JD, BG, AG and EMD providing critical feedback and editing to subsequent drafts. All authors read and approved the final manuscript.
Funding This work was supported by the Scottish Improvement Science Collaborating Centre (SISCC) which is funded by the Scottish Funding Council (SFC), Chief Scientist's Office, NHS Education for Scotland and The Health Foundation with in kind contributions from participating partner universities and health boards. grant number 242343290.

Competing interests None declared.

Patient consent for publication Not required.

Ethics approval The study was approved by the University Research Ethics Committee of the University of Dundee (REC reference number: 2016017_Toma) before any participant was approached.

Provenance and peer review Not commissioned; externally peer reviewed.

Data availability statement All data relevant to the study are included in the article or uploaded as online supplementary information. All materials used in this study can be found in the online supplemental material.

Open access This is an open access article distributed in accordance with the Creative Commons Attribution Non Commercial (CC BY-NC 4.0) license, which permits others to distribute, remix, adapt, build upon this work non-commercially, and license their derivative works on different terms, provided the original work is properly cited, appropriate credit is given, any changes made indicated, and the use is non-commercial. See: http://creativecommons.org/licenses/by-nc/4.0/.

ORCID iDs

Jason Tang http://orcid.org/0000-0002-2891-5507

Aileen Grant http://orcid.org/0000-0001-6146-101X

\section{REFERENCES}

1 Howard RL, Avery AJ, Slavenburg S, et al. Which drugs cause preventable admissions to hospital? A systematic review. Br J Clin Pharmacol 2007;63:136-47.

2 Pirmohamed M, James S, Meakin S, et al. Adverse drug reactions as cause of admission to hospital: prospective analysis of 18820 patients. BMJ 2004;329:15-19.

3 Lea M, Mowe M, Mathiesen L, et al. Prevalence and risk factors of drug-related hospitalizations in multimorbid patients admitted to an internal medicine ward. PLoS One 2019;14:e0220071.

4 Marlen Schurig A, Böhme M S, Just K, et al. Adverse drug reactions (ADR) and emergencies. Dtsch Arztebl Int 2018;115:251-8.

5 Leendertse AJ, Egberts ACG, Stoker LJ, et al. Frequency of and risk factors for preventable medication-related hospital admissions in the Netherlands. Arch Intern Med 2008;168:1890-6.

6 Guthrie B, McCowan C, Davey P, et al. High risk prescribing in primary care patients particularly vulnerable to adverse drug events: cross sectional population database analysis in Scottish general practice. BMJ 2011;342:d3514.

7 Dumbreck S, Flynn A, Nairn M, et al. Drug-disease and drug-drug interactions: systematic examination of recommendations in 12 UK national clinical guidelines. 350. British Medical Journal, 2015.

8 Guthrie B, Makubate B, Hernandez-Santiago V, et al. The rising tide of polypharmacy and drug-drug interactions: population database analysis 1995-2010. BMC Med 2015;13:74.

9 Burgess C, Holman C, Satti A. Adverse drug reactions in older Australians, 1981-2002. Med J Aust 2005;182.

10 Bourgeois FT, Shannon MW, Valim C, et al. Adverse drug events in the outpatient setting: an 11-year national analysis. Pharmacoepidemiol Drug Saf 2010;19:901-10.

11 Avery AJ, Rodgers S, Cantrill JA, et al. A pharmacist-led information technology intervention for medication errors (pincer): a multicentre, cluster randomised, controlled trial and cost-effectiveness analysis. Lancet 2012;379:1310-9.

12 Dreischulte T, Donnan P, Grant A, et al. Safer Prescribing--A Trial of Education, Informatics, and Financial Incentives. N Engl J Med 2016;374:1053-64.

13 Scottish Government Polypharmacy Model of Care Group. Polypharmacy guidance, realistic prescribing. 3rd edn. Scottish Government, 2018.

14 Hobbs FDR, Bankhead C, Mukhtar T, et al. Clinical workload in UK primary care: a retrospective analysis of 100 million consultations in England, 2007-14. The Lancet 2016;387:2323-30.

15 The pharmaceutical Journal 2018;300.

16 Health in Wales. clinical pharmacists in GP practice and primary care clusters. Available: http://www.wales.nhs.uk/news/40188 [Accessed 16 Oct 2018].

17 The pharmaceutical Journal, PJ 2015. 
18 NHS England. News. more than 400 pharmacists to be recruited to GP surgeries by next year, 2015. Available: https://www.england.nhs. uk/2015/11/pharmacists-recruited/

19 Michie S, Atkins L. The Behaviour Change Wheel - A Guide To Designing Interventions. 1th edn. Silverback Publishing, 2014.

20 Cane J, O'Connor D, Michie S. Validation of the theoretical domains framework for use in behaviour change and implementation research. Implement Sci 2012;7:37.

21 Phillips CJ, Marshall AP, Chaves NJ, et al. Experiences of using the theoretical domains framework across diverse clinical environments: a qualitative study. J Multidiscip Healthc 2015;8:139-46.

22 Cadogan CA, Ryan C, Francis JJ, et al. Improving appropriate polypharmacy for older people in primary care: selecting components of an evidence-based intervention to target prescribing and dispensing. Implement Sci 2015;10:161.

23 Michie S, Atkins L. Chapter 2 - Step 5: Identifying intervention functions. In: The behaviour change wheel. Silverback publishing, 2014.

24 Michie S, Atkins L. Chapter 2 - Step 6: Identifying policy categories. In: The behaviour change wheel. Silverback publishing, 2014.
25 Michie S, Atkins L. Chapter 3 - Step 7: Identifying behaviour change techniques. In: The behaviour change wheel. Silverback publishing, 2014.

26 Grant A, Dreischulte T, Guthrie B. Process evaluation of the data-driven quality improvement in primary care (DQIP) trial: case study evaluation of adoption and maintenance of a complex intervention to reduce high-risk primary care prescribing. BMJ Open 2017;7:e015281.

27 Grant A, Dreischulte T, Guthrie B. Process evaluation of the datadriven quality improvement in primary care (DQIP) trial: active and less active ingredients of a multi-component complex intervention to reduce high-risk primary care prescribing. Implement Sci 2017;12:4.

28 Craig P, Dieppe P, Macintyre S, et al. Developing and evaluating complex interventions: the new medical Research Council guidance. BMJ 2008;337:a1655.

29 Nkansah N, Mostovetsky O, Yu C, et al. Effect of outpatient pharmacists' non-dispensing roles on patient outcomes and prescribing patterns. Cochrane Database Syst Rev 2010;7:Cd000336. 